\title{
Gain-Switched Optical Frequency Combs for Future Mobile Radio-Over-Fiber Millimeter-Wave Systems
}

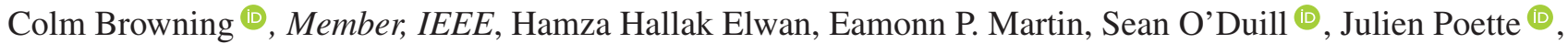 \\ Paul Sheridan, Arman Farhang ${ }^{\circledR}$, Member, IEEE, Beatrice Cabon, and Liam P. Barry ${ }^{\circledR}$, Senior Member, IEEE
}

\begin{abstract}
The millimeter-wave (mm-wave) frequency band has emerged as a means to overcome current radio frequency spectral limitations and represents an interesting solution to fulfill the bandwidth and networking requirements of fifth generation (5G) mobile communications and beyond. Photonic generation of these frequencies holds advantages over electronic methods in terms of cost and effective network distribution. Due to their coherent nature, optical frequency combs (OFC) are a promising solution for the efficient generation of $\mathrm{mm}$-wave frequencies. The work outlined examines the use of OFCs in a mm-wave radio-over-fiber (RoF) heterodyne system with regard to the specific requirements of a $5 \mathrm{G}$ candidate waveform, universally filtered orthogonal frequency division multiplexing. Through experimentation and simulation, the key limitations of linewidth, effective path length difference, and relative intensity noise (RIN) are explored. Results are presented, in terms of error vector magnitude (EVM), for a wide range of system parameters highlighting important considerations to be taken in designing future mm-wave RoF systems employing OFCs. Performance of $\sim 5 \%$ EVM using single sideband modulation is achieved for optimized system conditions and an RIN level of $-132 \mathrm{~dB} / \mathrm{Hz}$.
\end{abstract}

Index Terms-5G, fronthaul, millimeter wave communications, optical frequency combs, radio-over-fiber, UF-OFDM.

\section{INTRODUCTION}

$\mathbf{M}$ OBILE communications networks will be required to adapt in order to facilitate $5 \mathrm{G}$ and future mobile

Manuscript received February 15, 2018; revised April 17, 2018; accepted May 19, 2018. Date of publication May 28, 2018; date of current version August 30, 2018. This work was supported by the Irish Research Council and French Ministry of Foreign Affairs under the Ulysses Mobility programme. The publication has emanated from research supported in part by a research grant from Science Foundation Ireland and is co-funded under the European Regional Development Fund under Grant Numbers 13/RC/2077 and 12/RC/2276. (Corresponding author: Colm Browning.)

C. Browning, E. P. Martin, S. Doyle, P. Sheridan, and L. P. Barry are with the School of Electronic Engineering, Dublin City University, Dublin D09W6Y4, Ireland (e-mail: colm.browning@dcu.ie; eamonn.martin@dcu.ie; sean.oduill@dcu.ie; paul.sheridan25@mail.dcu.ie; liam.barry@dcu.ie).

A. Farhang is with the School of Electrical and Electronic Engineering, University College Dublin, Dublin 4, Ireland (e-mail: farhanga@tcd.ie).

H. H. Elwan, J. Poette, and B. Cabon are with the Grenoble Alpes University, Institute of Microelectronics Electromagnetism and Photonics and the Laboratory of Microwave and Characterization, 38000 Grenoble, France (e-mail: hamza.hallak-elwan@imep.grenoble-inp.fr; julien.poette@ grenobleinp.fr; beatrice.cabon@grenoble-inp.fr).

Color versions of one or more of the figures in this paper are available online at http://ieeexplore.iee.org.

Digital Object Identifier 10.1109/JLT.2018.2841365 communications. Greatly increased network speeds will be required, as well a fundamental shift in the make up these networks. This will include ultra-dense (UD) antenna deployment and vastly increased fiber penetration to these sites [1], which will be necessary in order to deliver the types of high speed services envisaged for the future connected society; virtual reality (VR) applications, 4/8K high definition (HD) streaming, live HD video editing etc.

The successful delivery of next generation services, to an ever-increasing number of connected users and devices, will depend highly on the available bandwidth in the wireless network. This may be extremely difficult to facilitate given current spectral occupancy. This has led to the emergence of mm-wave $(30-300 \mathrm{GHz})$ communications, which can provide large amounts of available bandwidth, and hence, extremely high channel capacity suitable for high data rate wireless transmission. These advantages have meant that mm-wave communications have come to be considered as a key enabling technology for $5 \mathrm{G}$ and beyond [2].

The effective utilization of $\mathrm{mm}$-wave frequencies for mobile communications depends on the efficiency, in terms of both cost and power, with which the carriers can be generated. Generation of mm-wave signals in the electronic domain is hampered by the cost and complexity of the components required and so photonic generation, i.e., optical heterodyne techniques, have been proposed [3], [4]. Aside from the economic advantages, the use of the optical domain for mm-wave signal generation naturally lends itself to increased optical distribution through fronthaul and mid-haul networks - key optical networking structures that enable increased centralization of resources and UD small cell distribution. Furthermore, optical heterodyning offers scalability as higher frequency generation can be easily facilitated through the use of tunable optical sources.

Mm-wave generation in this way has been demonstrated using many types of laser sources. Li et al. [5] employed two freerunning external cavity lasers (ECL) to achieve $60 \mathrm{~Gb} / \mathrm{s} \mathrm{RoF}$ transmission of 64-quadrature amplitude modulation (QAM) data, while Alavi et al. [6] presents the use of an extremely low linewidth dual wavelength fibre laser to demonstrate multicarrier RoF transmission suitable for $5 \mathrm{G}$ applications. However, the complexities associated with these methods [7] have contributed to the sustained interest in the use of OFCs as an 
economic and effective optical source enabling mm-wave RoF communications [8]-[11].

Two common methods to generate OFCs are; the use of mode locked laser (MLL) [12] diodes and optical gain-switching of a laser diode [13]. MLLs suffer from large linewidth $(\mathrm{MHz}$ range) and mode partition noise which can be imparted to the heterodyne mm-wave signal [14], and, unlike combs generated through gain-switching, they exhibit a fixed free spectral range (FSR) which limits flexibility in the generated mm-wave carrier. Our recent work, [7], has experimentally demonstrated the use of an OFC generated through gain-switching for mm-wave RoF transmission appropriate for $5 \mathrm{G}$ applications. It has highlighted the heightened importance of coherence between optical carriers given the relatively low baud rates associated with mobile transmission.

The work presented in this paper expands on [7] to provide an analysis, in terms of optimum operating conditions and system limitations, on the use of OFCs generated by gain-switching for mobile RoF mm-wave distribution. Our numerical simulations and experimental testbed results encompass key factors associated with optical heterodyning, namely; the impact of optical linewidth, effective path difference and relative intensity noise (RIN). This work analyzes the impact of those factors which are varied within the context of the implemented system to (i) find system limitations, (ii) identify optimum operating conditions and (iii) provide important design rules for the construction of futute RoF mm-wave systems employing OFCs. System performance is evaluated, for the various tested operating conditions, in terms of the received EVM of a $5 \mathrm{G}$ candidate waveform, universally filtered orthogonal frequency division multiplexing (UF-OFDM) which offers increased robustness to timing synchronization and reduced out-of-band (OOB) emission compared to conventional OFDM [15]. For optimum conditions EVMs of around 5\% are achieved for single sideband (SSB) transmission using a complex optical (I/Q) modulator.

The remainder of the paper is outlined as follows: Section II briefly describes the properties of the UF-OFDM waveform used throughout the simulation and experimental work. Section III describes the experimental setup and Section IV describes the numerical simulation undertaken. Section V includes discussion on the obtained performance with regard to the impact of optical linewidth, and the impact of RIN for various OFC configurations. Finally, Section VI outlines the conclusions of the work.

\section{UF-OFDM: A FIFTH GENERATION CANDIDATE WAVEFORM}

UF-OFDM is a multi-carrier modulation scheme which is considered to be a candidate waveform for 5G [16]. Like OFDM, its implementation is (inverse) fast Fourier transform ((I)FFT) based and involves the transmission of subcarriers, each typically modulated with QAM symbols. UF-OFDM lessens the strict orthogonality requirements imposed by OFDM, by employing linear filtering of sub-bands of the transmitted subcarriers. Subcarriers in each sub-band (or resource block) are orthogonal to each other, but not to subcarriers contained in other sub-bands. Linear filtering leads to a much lower OOB
TABLE I

UF-OFDM SIGNAL PROPERTIES

\begin{tabular}{|c|c|}
\hline Property & Value \\
\hline Bandwidth & $152 \mathrm{MHz}$ \\
\hline No. Subcarriers & 76 \\
\hline Subcarriers Baud Rate & $2 \mathrm{MHz}$ \\
\hline UF-OFDM sub-bands & 4 \\
\hline Raw Data Rate & $0.912 \mathrm{~Gb} / \mathrm{s}$ \\
\hline Modulation & 64-QAM \\
\hline
\end{tabular}

emission and this results in a relaxation of synchronization requirements [17]. Further discussion of UF-OFDM and other 'OFDM inspired' waveforms can be found in [16].

UF-OFDM bands with the properties outlined in Table I are used throughout this work for data modulation.

\section{EXPERIMENTAL SETUP}

The optical heterodyne mm-wave experimental setup is shown in Fig. 1. The system architecture is designed to replicate a mobile fronthaul link where analog RoF signals are transmitted from a central office (CO) through $25 \mathrm{~km}$ of standard single mode fiber (SSMF) to a remote radio head (RRH)-though no wireless transmission is performed.

The light source used is an OFC based on gain switchinghighlighted in green in Fig. 1. A $17.6 \mathrm{GHz}$ sinusoid is used to gain switch a distributed feed-back (DFB) laser resulting in multiple, coherent, optical tones with a FSR of $17.6 \mathrm{GHz}$ as shown in Fig. 2. An ECL is set up so that optical injection of the DFB may be performed. This offers a highly reduced comb linewidth at the expense of higher system cost/complexity [18] and is employed here for comparison with simulation results relating to the impact of the optical linewidth. A detailed investigation into the coherence of a $60 \mathrm{GHz}$ signal resulting from the beating of two partially phase uncorrelated optical tones generated using gain switched DFB may be found in [19]. A wavelength selective switch (WSS) is used to select two optical tones spaced by the required mm-wave frequency, in this case $52.8 \mathrm{GHz}$ (which is three times the FSR of the OFC). An erbium doped fiber amplifier (EDFA) is used to boost the power of the optical carriers before the optical path is split in two using a 50:50 coupler.

A Tektronix arbitrary waveform generator (AWG) operating at $20 \mathrm{GSa} / \mathrm{s}$ is used to generate five UF-OFDM bands at different RF carrier frequencies centered around $2.05 \mathrm{GHz}$ and with a $10 \mathrm{MHz}$ guard band between each. Each band was constructed with the properties outlined in Table I. One of the selected optical tones from the OFC undergoes carrier suppressed single sideband (CS-SSB) modulation using an electrical 90 degree hybrid coupler and a complex (I/Q) optical modulator as illustrated in Fig. 1. A tunable optical delay line is inserted in the unmodulated tone's path. This is to allow the phase offset between the two optical paths (due to path length difference including the dispersive effect of the SSMF) to be pre-compensated for 


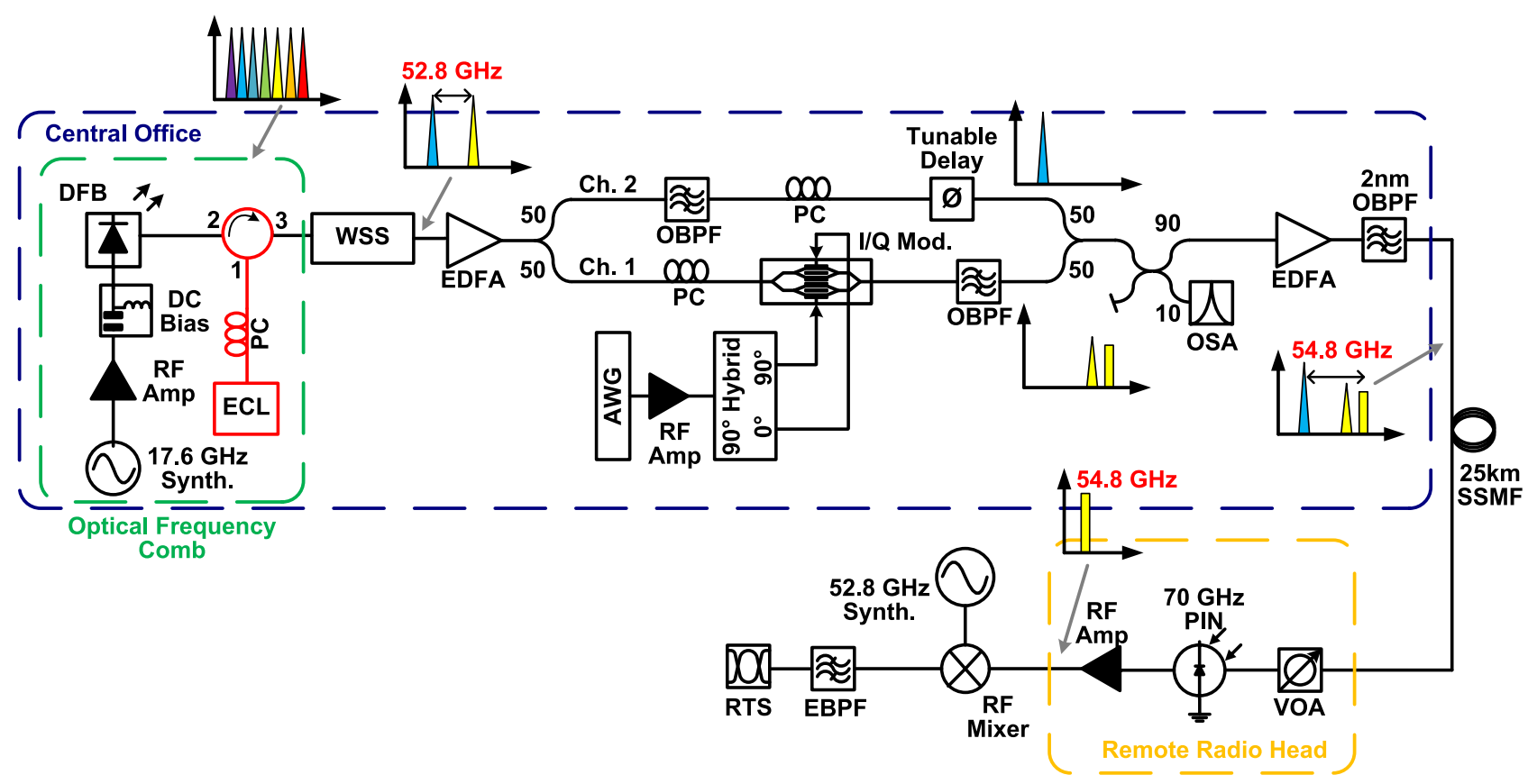

Fig. 1. Experimental heterodyne RoF mm-wave setup including figurative spectral representations of the heterodyne process. The setup is designed to resemble an optical front-haul network eith consolidated equipment at the Central Office, and analog RoF transmission to a relatively simplistic Remote Radio Head.

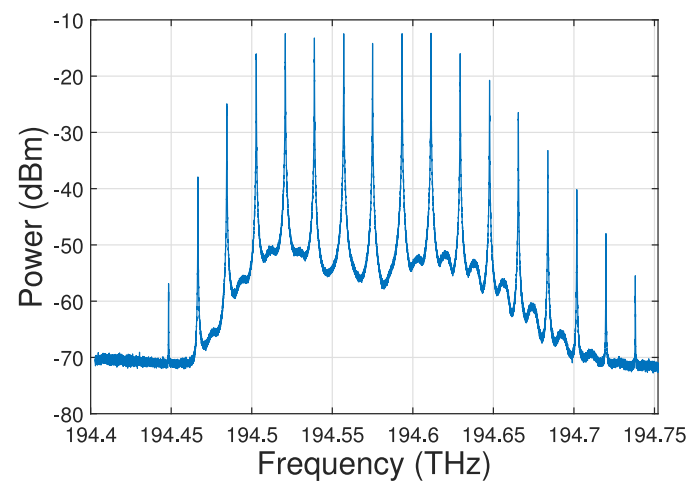

Fig. 2. Experimentally measured OFC from a gain switched DFB laser, with an FSR of 17.6 GHz. Operating conditions: $58 \mathrm{~mA}$ bias current, $22 \mathrm{dBm}$ RF drive.

optimum performance [7], [11], and swept to observe system limitations.

The modulated and un-modulated tones are both passed through an optical bandpass filter (OBPF) to increase the rejection of adjacent optical comb tones. The tones are then coupled together, amplified to $7 \mathrm{dBm}$ using an EDFA and transmitted through the fiber. At the RRH side, a variable optical attenuator (VOA) is used to restrict the input power to the $70 \mathrm{GHz}$ PIN photo-diode to $0 \mathrm{dBm}$. The optical components undergo photomixing on the diode with the term of interest resulting from the beating of the un-modulated optical tone and the UF-OFDM data bands - produced at $54.8 \mathrm{GHz}$. This mm-wave signal is amplified with a $60 \mathrm{GHz}$ RF amplifier, transmitted through a $50 \mathrm{~cm}$ long waveguide and mixed down to the original RF frequencies using a $52.8 \mathrm{GHz}$ local oscillator (LO). The electrical signal is band-pass filtered and captured using a Tektronix real time oscilloscope (RTS) sampling at $50 \mathrm{GSa} / \mathrm{s}$. De-modulation and EVM calculations are performed off-line.

\section{NUMERICAL SimULATION}

The constructed system simulator is designed in Matlab and closely resembles the experimental system shown in Fig. 1. This way, the impacts on the system performance of the comb source phase noise, and relative path delay between the modulated and un-modulated carriers which beat on the photo-diode to generate the mm-wave data, can be modeled. To simulate the gain-switched comb source with $17.6 \mathrm{GHz}$ FSR, we numerically create an idealized comb source with nine equal-amplitude comb tones. The condition for creating a frequency comb is that each comb tone has exactly the same (i.e., correlated) phase noise with a specified linewidth broadening of the tones. Therefore we can write the optical field for the comb as

$$
\left(\sum_{k=-4}^{4} \sqrt{1+n_{\mathrm{RIN}}} \cdot E_{k} \exp \left(j 2 \pi k \Delta f_{\mathrm{FSR}} t\right)\right) \exp (\varphi(t))
$$

where $E_{\mathrm{k}}$ represents the optical field amplitude of the $k$ th comb tone, therefore $\left|E_{k}\right|^{2}$ represents the optical power of the $k t h$ comb tone. $\Delta f_{\mathrm{FSR}}$ is the FSR of the comb source and taken to be 17.6 GHz throughout. The phase noise of the comb is contained within the term $\varphi(t)$. The calibrated random-walk phase noise can be generated by firstly generating $\hat{f}(m)$ taking $M$ samples (one for each sampling point in the entire signal) taken from a Gaussian distributed randomly generated waveform with zeromean and unity variance. The random walk phase noise at the $l$ th time step is then implemented by taking a scaled running 


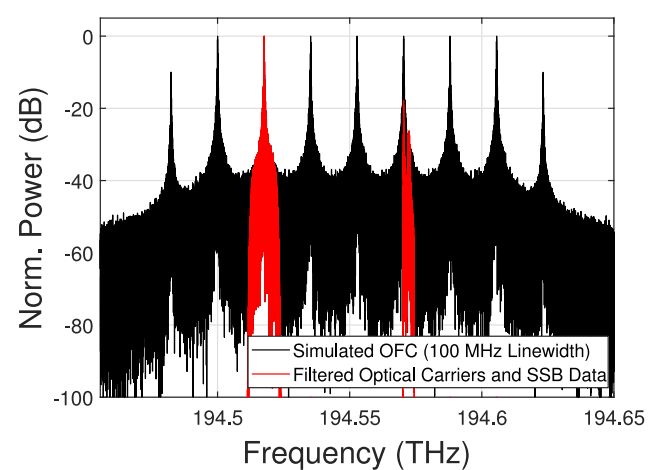

Fig. 3. Simulated comb of ideal carrier with added arbitrary linewidth, filtered tones and data (in red).

sum of $\hat{f}(m),[20]$,

$$
\varphi(l)=\sqrt{2 \pi B_{\mathrm{o}} t_{\mathrm{s}}} \sum_{m=0}^{l} \hat{f}(m)
$$

where $B_{\mathrm{o}}$ is the $3 \mathrm{~dB}$ linewidth and $t_{\mathrm{s}}$ the signal sampling period - taken to be 1 ps throughout the simulation. $n_{\mathrm{RIN}}$ represents the RIN of the OFC and is generated by taking

$$
n_{\mathrm{RIN}}=h_{\mathrm{LPF}} \otimes \sqrt{R I N_{\mathrm{lin}} / t_{\mathrm{s}}} \cdot e_{\mathrm{n}}
$$

where $e_{\mathrm{n}}$ are the samples taken from a unity variance, zeromean Gaussian distribution, $R I N_{\text {lin }}$ is the the value of $R I N_{\mathrm{dB}}$ in linear terms. These samples are band limited by convolution with a $10 \mathrm{GHz}$ low pass filter with the impulse response $h_{\mathrm{LPF}}$.

As in the experiment, two comb tones separated by 3 $\times \Delta f_{\mathrm{FSR}}$ are filtered out. We numerically implement Gaussianshaped OBPFs to filter two individual comb tones $E_{\mathrm{c} 1}$ and $E_{\mathrm{c} 2}$ spaced by $52.8 \mathrm{GHz}$ - achieving the functionality of the WSS in the experimental system. Fig. 3 shows the simulated optical comb with $100 \mathrm{MHz}$ linewidth (in black) and the filtered tones (one with CS-SSB modulation) separated by the mm-wave frequency of $52.8 \mathrm{GHz}$ (red). The variable delay is implemented by circularly shifting the numerical array representing the optically band-passed field of the un-modulated comb tone by a number of samples points that correspond to the chosen delay $T_{\mathrm{d}}$. The other comb tone is modulated by driving a complex I/Q modulator with the same UF-OFDM waveform outlined in Section II. The biasing conditions of the simulated modulator are set so that the optical field of $E_{\mathrm{c} 2}$ is modulated according to the field of the driving signal, and SSB modulation of the optical carrier is achieved. The un-modulated and modulated comb tones are then combined to form the composite optical signal. Fiber transmission through $25 \mathrm{~km}$ of SSMF was simulated by modeling the propagation of the electric field using the nonlinear Schrödinger equation (NLSE), which was solved using the split step Fouier method for ten steps per km over the length. Photo-mixing of the optical components occurs at the modeled high speed photo-diode to create an electrical mm-wave $5 \mathrm{G}$ signal (carrier at $52.8 \mathrm{GHz}$ ). The electrical photo-current is generated as:

$$
I_{\mathrm{pd}}=R_{\mathrm{pd}}\left|E_{\mathrm{c} 1}\left(t-T_{\mathrm{d}}\right)+E_{\mathrm{c} 2}(t)\right|^{2}
$$

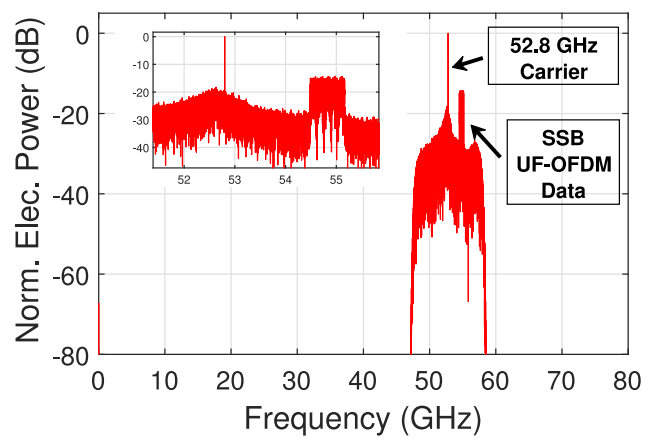

Fig. 4. Simulated generated mm-wave carrier and SSB OF-OFDM data bands.

where $R_{\mathrm{pd}}$ is the responsivity in $\mathrm{A} / \mathrm{W}$ of the photo-diode. We add the shot noise generated by the photo-diode with power spectral density:

$$
I_{\mathrm{sh}}=2 q\left\langle I_{\mathrm{pd}}\right\rangle .
$$

We can ignore the DC and low-frequency terms due to the RF band-pass filtering of the modulated signal generated at $52.8 \mathrm{GHz}$. The detected photo-current of the mm-wave signal centered at $52.8 \mathrm{GHz}$ is effectively given by:

$$
i_{\mathrm{RF}}(t)=R_{\mathrm{pd}} E_{\mathrm{c} 1}^{*}\left(t-T_{\mathrm{d}}\right) E_{\mathrm{c} 2}(t)
$$

We can see immediately from $i_{\mathrm{RF}}(t)$ that the residual phase noise transfer from the optical comb tones to $i_{\mathrm{RF}}(t)$ can be written as:

$$
\varphi_{\mathrm{RES}}(t)=\varphi(t)-\varphi\left(t-T_{d}\right) .
$$

Therefore, if the path delay difference for the two correlated comb tones is zero, then the phase noise of the mm-wave signal generated through photo-mixing is essentially zero, and hence optimum system performance is achieved. Though, when the delay between the two paths is not zero, the phase variance of the difference between the phase terms is by definition, [21],

$$
\left\langle\left[\varphi(t)-\varphi\left(t-T_{\mathrm{d}}\right)^{2}\right\rangle=2 \pi B_{\mathrm{o}}\left|T_{\mathrm{d}}\right| .\right.
$$

Therefore, the time delay between the optical tones reduces the level of correlation between them leading to additional phase noise on the generated RF signals - thus degrading system performance. Fig. 4 shows the simulated generated mm-wave carrier and data where there is no inserted time delay, $T_{\mathrm{d}}=0$ (i.e., perfect correlation between optical tones). From the zoomed inset, the 'delta function-like' carrier can be observed as this condition results in zero phase noise transfer to the mmwave carrier generated by the heterodyne technique.

The complete simulator allows us to understand the system performance by being able to adjust the system parameters such as the linewidth $B_{\mathrm{O}}$ and RIN level $n_{\mathrm{RIN}}$ of the comb tones as well as the effective path delay $T_{\mathrm{d}}$ between the two filtered comb tones. Excellent matching is achieved when the simulator is set to match the experimental conditions under test, as shown in the following sections. 


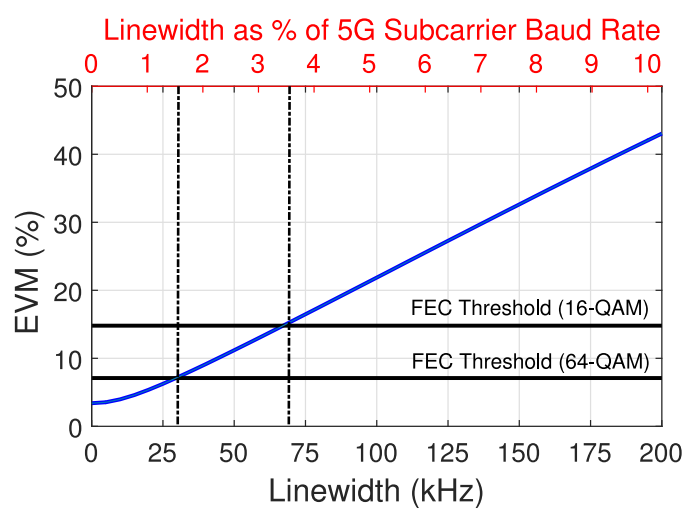

Fig. 5. Linewidth versus EVM when uncorrelated light sources are used for heterodyning in the simulated mm-wave RoF system.

\section{RESUlTS AND Discussion}

\section{A. Impact of Source Linewidth}

For mobile communications typically employing multicarrier modulation with low subcarrier baud rates, potentially less than $1 \mathrm{MHz}$, the photonic generation of a mm-wave carrier with sufficiently low phase noise will be of paramount importance.

Considering the use of independent laser sources (i.e., laser sources with uncorrelated phase noise) in a heterodyne system; the phase noises from both lasers will add and the linewidth of the mm-wave carrier will be the sum of the linewidths of the two optical sources. The fact that the optical sources are uncorrelated places a tight restriction on linewidth - in order to produce a mm-wave carrier with tolerably low phase noise. In the simulation, two independent optical sources were modeled by restricting the optical carrier number to two and generating both with the same $3 \mathrm{~dB}$ linewidth, $B_{o}$, though both sources having uncorrelated phase noise. Fig. 5 shows the overall simulated system performance in terms of average received EVM versus linewidth when two independent laser sources are used in the mm-wave heterodyne system. The figure shows that for the implemented system, with a moderate UF-OFDM subcarrier baud rate of $2 \mathrm{MHz}$, optical sources with a linewidth of $<30$ and $<70 \mathrm{kHz}$ are required for performance on the 64 and 16-QAM forward error correction (FEC) thresholds, respectively. These linewidths corresponds to $1.5 \%$ and $3.5 \%$ of the subcarrier baud rate used (shown on the upper $\mathrm{x}$-axis in the figure), respectively. Considering the requirements for a 64-QAM multi-carrier scheme; for subcarrier baud rates of $\geq 1 \mathrm{MHz}$, two independent ECLs (typical linewidth 10-20 kHz) may provide the necessary linewidth and tunability required for flexible mm-wave signal generation. However, for mobile systems employing lower subcarrier baud rates with higher order modulation, ECLs are incapable of providing the sub-kHz linewidth necessary. This fact, coupled with their bulkiness and expense will limit the feasibility of their implementation in future mmwave systems for mobile communications.

For transmitters employing coherent optical carriers, such as an OFC, total cancellation of the mm-wave beat tone's residual

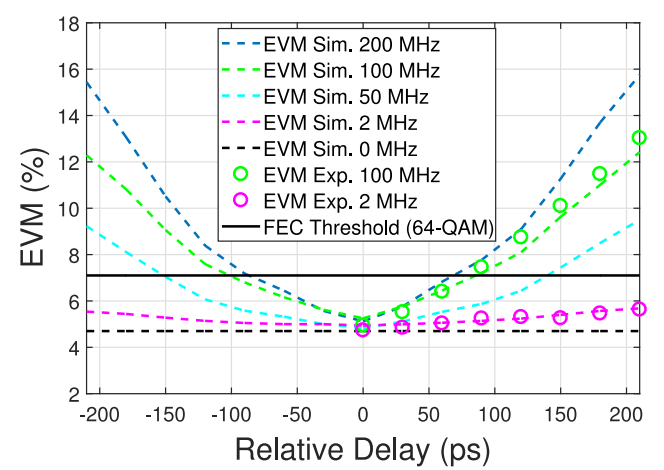

Fig. 6. EVM versus relative delay for various linewidths (experimental and simulation).

phase noise can be achieved provided that the optical carriers experience the same path delay through the system (as indicated by equation (7)). Our previous work has explored the use of effective path length pre-compensation at the transmitter side to ensure maximum correlation between optical carriers in a mm-wave heterodyne system employing $\mathrm{MHz}$ linewidth OFCs [7], [11]. Here, we analyze the performance of the constructed system for varying effective path length differences between the optical carriers, with regard to their linewidth.

Fig. 6 shows experimental and simulated system performance when a relative delay between the optical carriers over a range of \pm 210 ps (restricted to 0-210 ps in experimental testbed) is introduced. Clearly, optimum performance is achieved for a relative delay of 0 ps where both simulated and experimentally received UF-OFDM signals exhibit an average EVM of $\sim 5 \%$, irrespective of OFC linewidth. The figure shows that the extent to which performance is degraded, as relative delay increases, depends on the linewidth of OFC comb tones. This can be explained as the linewidth of the tones is inversely proportional to their coherence time, and hence their correlation with respect to a given delay, $T_{\mathrm{d}}$. This leads to the parabolic relationships shown in Fig. 6. A delayed self-heterodyne technique [22] was used to experimentally measure the linewidth of the OFC used (see Fig. 2) which was found to be $100 \mathrm{MHz}$. When the comb undergoes external injection from the ECL - for comparative reasons - the linewidth reduces to $2 \mathrm{MHz}$. It is important to note that, the OFC linewidth achieved when optical injection is performed is highly dependent on the injection conditions, such as power and wavelength detuning [23]. The master ECL in this case had a linewidth of approximately $16 \mathrm{kHz}$.

The figure highlights a clear trade-off between the use of higher linewidth OFCs, and the tolerance to effective path length differences in the system (due to either transmitter/receiver design or fiber dispersion). Excellent matching between experimental and simulated results is exhibited with average EVM performance reaching the 64-QAM FEC threshold for a relative delay of about $90 \mathrm{ps}$ in the $100 \mathrm{MHz}$ linewidth cases. This represents an effective optical path difference of less than $2 \mathrm{~cm}$. EVMs for the $2 \mathrm{MHz}$ linewidth cases do not exceed $6 \%$ over the tested delay range, providing a system margin before encountering the FEC threshold. 


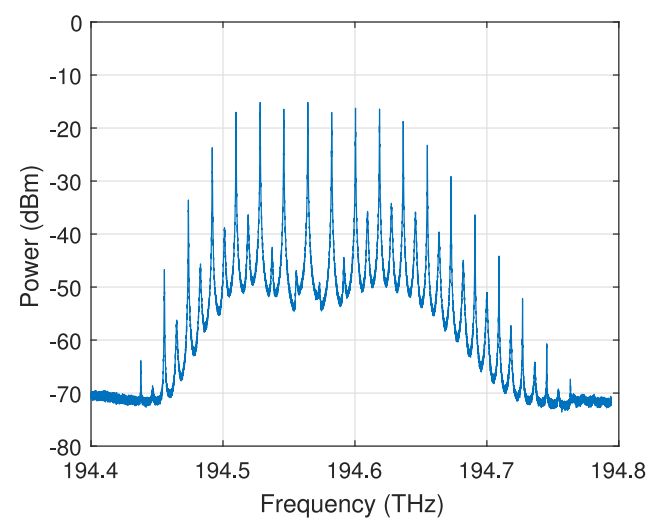

(a)

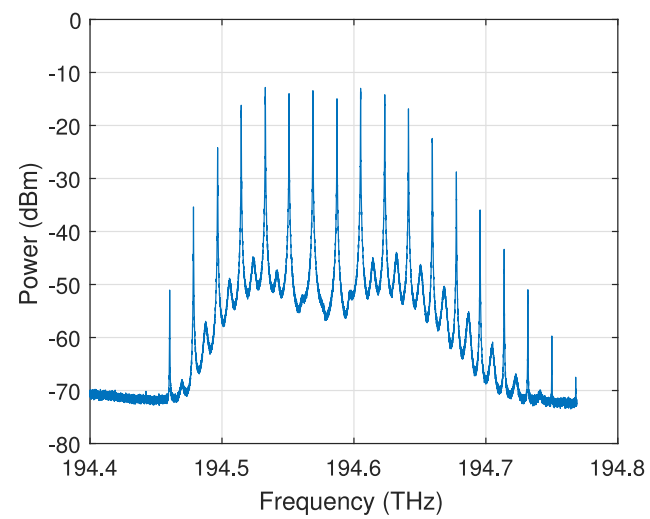

(b)

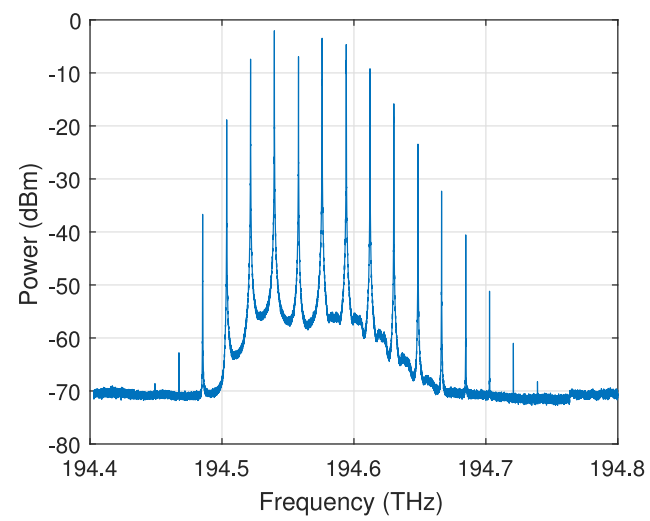

(c)

Fig. 7. Optical spectra OFCs generated under various biasing and RF drive signal conditions. (a) $50 \mathrm{~mA}$ bias current, $23.5 \mathrm{dBm} \mathrm{RF}$ drive, (b) $50 \mathrm{~mA}$ bias current, $22 \mathrm{dBm}$ RF drive, (c) $62 \mathrm{~mA}$ bias current, $19 \mathrm{dBm}$ RF drive.

\section{B. Impact of Relative Intensity Noise}

$\mathrm{RIN}$ is a random fluctuation of the laser's intensity with respect to time. Our previous work has shown that the RIN transferred to the generated mm-wave tone, as a result of optical hererodyning, impairs the performance of $60 \mathrm{GHz}$ RoF systems [24]. A detailed investigation of the impact of other amplitude noise contributions can be found in [25].

In order to study the effects of RIN in this experimental system, four OFCs were generated (shown in Figs. 2 and 7) by using four sets of operating conditions; namely laser bias current and the power of the RF drive signal used to perform gain

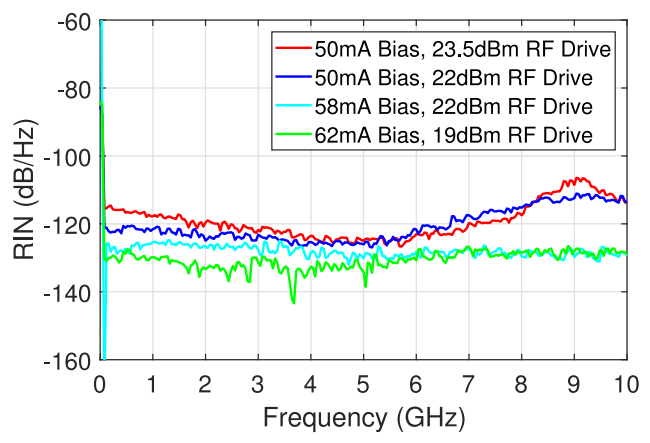

Fig. 8. Measured RIN profiles for the OFCs shown on Figs. 2 and 7.

switching (given in the captions in Figs. 2 and 7). Fig. 8 shows the experimentally measured RIN profiles of one tone from each tested OFC. Comb tones of the same OFC with similar power levels exhibit similar RIN profiles. Both the bias current and RF drive power affect the 'depth' of gain switching (i.e., the proximity of the RF drive signal to the laser threshold current). This results in OFCs with a varying number of comb tones and, importantly, levels of RIN, as shown by Fig. 7 and corresponding profile in Fig. 8. The greater the depth of gain switching, the greater number of optical tones are generated, with a higher level of RIN (and vice-versa).

This presents another interesting system trade off. At the expense of increased RIN, a greater number of tones are attained allowing flexibility in terms of; i) the tones which can be selected for heterodyning and ii) the facilitation of increased network functionality, by using the same OFC to provide optical carriers for multiple mm-wave heterodyne receivers. It should also be mentioned that OFC 'flatness' (i.e., the amount of comb tones within a given power range) is often used to assess the quality of optical combs, and depends greatly on the aforementioned laser driving conditions. The requirement for comb flatness, for heterodyne systems such as the one proposed in this work, is lessened due to the fact that just two comb tones, spaced by the desired mm-wave, are selected for heterodyning. This widens the range of operating conditions which can be successfully employed as evidenced by the following results.

Fig. 8 not only shows how RIN levels change for the driving conditions but also how the profile changes with respect to frequency. Over the relatively small bandwidth of the transmitted UF-OFDM bands (centered around $2 \mathrm{GHz}$ ), the RIN profile can be approximated as flat, and this is the approach taken in the simulation. However, Fig. 8 shows how the placement of the data bands hold importance for system performance as, for the OFCs tested, RIN levels between the frequencies of 2 and $4 \mathrm{GHz}$ vary by approximately $4-5 \mathrm{~dB} / \mathrm{Hz}$. By the same token, maximizing data throughput by increasing signal bandwidths must be carried out while remaining cognizant of the source RIN profiles in order to maintain sufficient system performance, as investigated in [24].

Fig. 9 shows the experimental ((a), (b)) and simulated ((c)-(d)) received constellation diagrams, and corresponding EVMs of the first UF-OFDM band (centered at $1.7 \mathrm{GHz}$ from the mm-wave carrier), for the OFCs exhibiting the lowest and 


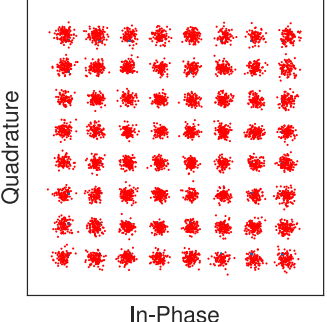

(a)

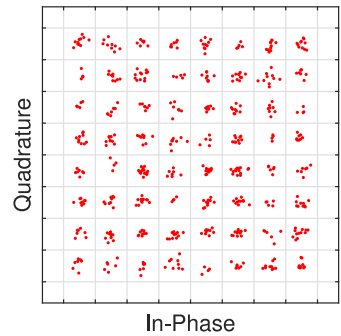

(c)

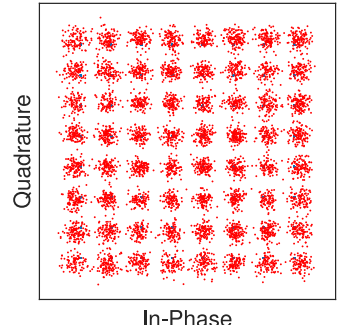

(b)

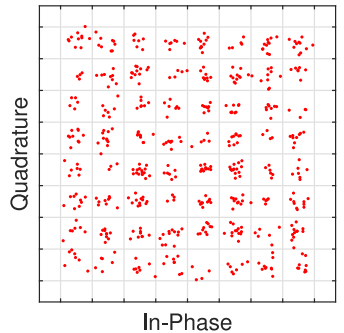

(d)
Fig. 9. Received experimental ((a),(b)) and simulated((c),(d)) constellations for the UF-OFDM band centered at $1.7 \mathrm{GHz}$ from the carrier. (a) $5.4 \% \mathrm{EVM}$, RIN: $-132 \mathrm{~dB} / \mathrm{Hz}$, (b) $7.7 \%$ EVM, RIN: $-115 \mathrm{~dB} / \mathrm{Hz}$, (c) $5.2 \%$ EVM, RIN: $-132 \mathrm{~dB} / \mathrm{Hz}$, (d) $7.4 \%$ EVM, RIN: $-115 \mathrm{~dB} / \mathrm{Hz}$.

highest levels of RIN. In all cases, the OFC was free running (i.e., un-injected) and effective path lengths were matched for optimum performance. The experimental performances of $5.4 \%$, $6.2 \%, 7.1 \%$ and $7.7 \%$ EVM were in line with the corresponding RIN measurements at the test frequency: $-132,-125,-120$ and $-115 \mathrm{~dB} / \mathrm{Hz}$, respectively. Similar performances are attained by simulation (examples in Fig. 9(c) and (d)) where a smaller number of transmitted symbols were employed in order to reduce computational complexity. Again, these results indicate excellent matching between the constructed simulator and the experimental testbed.

\section{CONCLUSION}

The exploitation of available free spectrum and proliferation of fiber connections to small cell sites have been identified as key requirements for future networks. This will make the bandwidth and services envisaged for $5 \mathrm{G}$, and future mobile communications, a reality. Photonic based mm-wave communications can help to satisfy these requirements by handling mobile data transport efficiently through the synergy of the electronic and optical domains. For future mobile mm-wave RoF systems employing heterodyning, OFCs hold great potential as they provide a cost effective and flexible means for the generation of suitable optical carriers.

An optical heterodyne RoF mm-wave simulator has been developed and excellent matching between simulated and experimental results is obtained. In both the experimental and numerical systems, the transmission of five bands of UF-OFDM, with an aggregate data rate of $4.56 \mathrm{~Gb} / \mathrm{s}$, using a complex optical modulator to achieve SSB modulation, is successfully demonstrated.
This work has shown that coherence between the transmitted optical carriers is a highly advantageous feature of OFCs for these systems, specifically considering the subcarrier baud rates associated with mobile multi-carrier modulation schemes. The work has highlighted the strict, and potentially inhibiting, linewidth requirements placed on uncorrelated optical sources for use in future heterodyne systems designed to transmit multicarrier mobile modulation formats. Furthermore, the analysis of the impact of RIN has indicated a trade-off, in these systems, between overall system performance and the potential for increased networking flexibility. This serves to inform important design rules for the future development and deployment of OFC based heterodyne mm-wave communication systems. Through the results obtained, and the analysis provided, the paper presents a thorough examination of the limitations and trade-offs associated with the practical implementation of these systems.

\section{REFERENCES}

[1] J. G. Andrews et al., "What will 5G be?" IEEE J. Sel. Commun., vol. 32, no. 6, pp. 1065-1082, Jun. 2014

[2] M. Xiao et al., "Millimeter wave communications for future mobile networks," IEEE J. Sel. Areas Commun., vol. 35, no. 9, pp. 1909-1935, Sep. 2017.

[3] A. Caballero, D. Zibar, R. Sambaraju, N. G. Gonzalez, and I. T. Monroy, "Engineering rules for optical generation and detection of high speed wireless millimeter-wave band signals," in Proc. 37th Eur. Conf. Opt. Commun., Genoa, Italy, 2011, pp. 1-3.

[4] R. Puerta, J. Yu, X. Li, Y. Xu, J. J. Vegas Olmos, and I. Tafur Monroy, "Single-carrier dual-polarization $328 \mathrm{~Gb} / \mathrm{s}$ wireless transmission in a D-band millimeter wave $2 \times 2$ MU-MIMO radio-over-fiber system," $J$. Lightw. Technol., vol. 36, no. 2, pp. 587-593, Jan. 2017.

[5] X. Li et al., "60-Gbps W-Band 64QAM RoF system with T-spaced DDLMS equalization," in Proc. Opt. Fiber Commun. Conf. Exhib., 2017, pp. 1-3.

[6] S. E. Alavi, M. R. K. Soltanian, I. S. Amiri, M. Khalily, A. S. M. Supa'at, and H. Ahmad, "Towards 5G: A photonic based millimeter wave signal generation for applying in 5G access fronthaul," Sci. Rep., vol. 6, 2016, Art. no. 19891

[7] C. Browning, E. P. Martin, A. Farhang and L. P. Barry, "60 GHz 5G radioover-fiber using UF-OFDM with optical heterodyning," IEEE Photon. Technol. Lett., vol. 29, no. 23, pp. 2059-2062, Dec. 2017.

[8] S. Koenig et al., "Wireless sub-THz communication system with high data rate," Nat. Photon., vol. 7, pp. 977-981, 2013.

[9] A. Stohr et al., "Millimeter-Wave photonic components for broadband wireless systems," IEEE Trans. Microw. Theory Techn., vol. 58, no. 11, pp. 3071-3082, Nov. 2010

[10] B. A. Khawaja and M. J. Cryan, "Wireless hybrid mode locked lasers for next generation radio-over-fiber," J. Lightw. Technol., vol. 28, no. 16, pp. 2268-2276, Aug. 2010.

[11] T. Shao et al., "Chromatic dispersion-induced optical phase decorrelation in a $60 \mathrm{GHz}$ OFDM-RoF system," IEEE Photon. Technol. Lett., vol. 26, no. 20, pp. 2016-2019, Oct. 2014.

[12] F. Brendel et al., "Low-cost analog fiber optic links for in-house distribution of millimeter-wave signals," Int. J. Microw. Wireless Technol., vol. 3, pp. 231-236, Apr. 2011.

[13] E. P. Martin, V. Vujicic, P. Anandarajah, C. Browning, R. Llorente, and L. P. Barry, " $25 \mathrm{~Gb} / \mathrm{s}$ OFDM $60 \mathrm{GHz}$ radio over fibre system using an externally injected gain switched distributed feedback laser," in Proc. Eur. Conf. Opt. Commun., Cannes, France, 2014, pp. 1-3.

[14] H. H Elwan, R. Khayatzadeh, T. Shao, J. Poette, B. Cabon, and L. P. Barry, "Impact of laser mode partition noise on optical heterodyning at millimeter-wave frequencies," J. Lightw. Technol., vol. 34, no. 18, pp. 4278-4284, Sep. 2016.

[15] C. Browning et al., "5G wireless and wired convergence in a passive optical network using UF-OFDM and GFDM," in Proc. IEEE Int. Conf. Commun. Workshops, 2017, pp. 386-392. 
[16] B. Farhang-Boroujeny and H. Moradi, "OFDM inspired waveforms for 5G,” IEEE Commun. Surveys Tuts., vol. 18, no. 4, pp. 2474-2492, Fourth Quarter 2016.

[17] V. Vakilian, T. Wild, F. Schaich, S. ten Brink, and J. F. Frigon, "Universalfiltered multi-carrier technique for wireless systems beyond LTE," in Proc. IEEE Globecom Workshops, 2013, pp. 223-228.

[18] P. M. Anandarajah et al., "Flexible optical comb source for super channel systems," in Proc. Opt. Fiber Commun. Conf. Expo. Nat. Fiber Optic Eng. Conf., Anaheim, CA, USA, 2013, pp. 1-3.

[19] T. Shao, M. Beltrn, R. Zhou, P. M. Anandarajah, R. Llorente, and L. P. Barry, "60 GHz radio over fiber system based on gain-switched laser," $J$. Lightw. Technol., vol. 32, no. 20, pp. 3695-3703, Oct. 15, 2014.

[20] D. Marcuse, "Computer simulation of FSK laser spectra and of FSK-toASK conversion," J. Lightw. Technol., vol. 8, no. 7, pp. 1110-1122, Jul. 1990.

[21] K. Kikuchi, "Characterization of semiconductor-laser phase noise and estimation of bit-error rate performance with low-speed offline digital coherent receivers," Opt. Express, vol. 20, no. 5, pp. 5291-5302, 2012.

[22] T. Okoshi, K. Kikuchi, and A. Nakayama, "Novel method for high resolution measurement of laser output spectrum," Electron. Lett., vol. 16, pp. 630-631, 1980.

[23] R. Zhou, "Optical frequency comb source for next generation access networks," Ph.D. dissertation, Dublin City Univ., Dublin, Ireland, 2014, pp. 87-88.

[24] H. H. Elwan, R. Khayatzadeh, J. Poette, and B. Cabon, "Impact of relative intensity noise on $60-\mathrm{GHz}$ radio-over-fiber wireless transmission systems," J. Lightw. Technol., vol. 34, no. 20, pp. 4751-4757, Oct. 2016.

[25] R. Khayatzadeh, H. H. Elwan, J. Poette, and B. Cabon, "Impact of amplitude noise in millimeter-wave radio-over-fiber systems," J. Lightw. Technol., vol. 33, no. 13, pp. 2913-2919, Jul. 2015.

Colm Browning received the Bachelor in the Art of Engineering degree (hons.) from Trinity College Dublin, Dublin, Ireland, in 2009 in electronic engineering and a minor in mathematics (BA). He received the Ph.D. degree in September 2013 from the Radio and Optical Communications Laboratory, Dublin City University (DCU), Dublin, Ireland, where his research focussed on reconfigurable passive optical networks. His subsequent postdoctoral work continued collaborative work between DCU, Columbia University, and the University of Arizona in the field of agile optical networks for datacenter applications. $\mathrm{He}$ is currently working as a Research Fellow with DCU, where his main interests include radio-over-fibre, microwave photonic networking, and flexible access/short reach optical communications. In 2014, he was awarded a Fulbright scholarship to undertake postdoctoral research in Columbia University, New York, NY, USA, to investigate the use of fast tunable lasers in dynamic amplified optical networks.

Hamza Hallak Elwan received the B.Sc. degree in electronics and communications engineering from Aleppo University, Aleppo, Syria, in 2010. He received the Master's degree in electronics engineering and the Engineer Diploma degree in communications system engineering in a double degree program from Politecnico di Torino, Torino, Italy and Institut Polytechnique de Grenoble (Grenoble-INP) Ensimag, Grenoble, France, in 2014. He received the Ph.D. degree from IMEP-LAHC Laboratory and Grenoble-INP, Grenoble, France, in 2017. He is currently a Postdoctoral Researcher with XLIM Laboratory and Universit de Limoges, Limoges, France. His research fields include microwavephotonics, radio-over-fiber, optical systems, and MIMO photonic radar.

Eamonn P. Martin received the B.Eng. and M.Eng. degrees from Dublin City University (DCU), Dublin, Ireland, in 2009 and 2010, respectively. He received the Ph.D. degree from Radio and Optical Communications Lab, DCU, in May 2015. He is currently a Postdoctoral Researcher with the Radio and Optical Communications Lab, where he has predominately worked with optical frequency combs for the generation of millimeter-waves for distribution in $5 \mathrm{G}$ radio over fiber systems. His current research interests include the use optical frequency combs for not only millimeter-wave generation and radio over fibre distribution systems but also for optical sensing applications.
Sean O'Duill received the B.E. and Ph.D. degrees from the Department of Electronic Engineering, University College Dublin, Dublin, Ireland. The main topics of his doctoral research were on the characterization of widely tunable lasers for dual format signals with information encoded in amplitude and frequency of the laser beam and on the nonlinear transmission impairments of those same signals. From May 2008 until September 2011, he was a Postdoctoral Fellow with the Department of Electrical Engineering, Technion, Israel Institute of Technology, and was awarded a Lady Davis Postdoctoral Fellowship in September 2009. At the Technion, he was engaged in all-optical signal processing in novel quantum dash optical amplifiers, application of slow and fast light techniques for microwave-signal processing, and optical comb generation for $1.6 \mathrm{Tbit} / \mathrm{s}$ optical superchannels. In October 2011, he moved to the Institute for Photonics and Quantum-Electronics, Karlsruhe Institute of Technology (KIT), Karlsruhe, Germany. While at KIT, he was leading workpackage 4 of the FP7 project ERMES, where he developed a numerical framework to develop and understand the operation and optimization of directly modulated long-cavity lasers for optical access networks. Since November 2012, he has been with the Radio and Optical Communications Laboratory, Rince Institute, Dublin City University, Dublin, Ireland, concentrating mainly on simulating stochastic (random) processes to evaluate the performance of optical devices and communication systems.

Julien Poette received the Engineer Diploma degree from the French Engineering School ENSSAT (National School for Applied Science and Technologies) Lannion, France, in 2002, with a specialization in optoelectronics and a Research Master degree in science and techniques of telecommunication. He received the Ph.D. degree in physics in 2005 from Rennes 1 University, Rennes, France. Since 2008, he has been with the IMEP-LAHC Laboratory and is currently an Associate Professor with the National Polytechnics Institute of Grenoble, Grenoble, France. His research interests include high speed optoelectronic systems and optical frequency generation.

Paul Sheridan received the B.Sc. degree in applied physics from the Dublin Institute of Technology, Dublin, Ireland, in 2007. Following his degree, he held various technical and engineering roles in the photonics industry in Ireland. In August 2016, he decided to return to academia to pursue his Ph.D. working on the development of photonic subsystems for future inter and intradata center network connections. His research focuses on the use of fast switching tuneable lasers, and optical frequency combs in reconfigurable optical networks employing coherent optical transmission.

Arman Farhang received the B.Sc. degree in telecommunications engineering from the Azad University of Najafabad, Najafabad, Iran, in 2007, the M.Sc. degree in telecommunications engineering from the Sadjad University of Technology, Mashhad, Iran, in 2010, and the Ph.D. degree from the Trinity College Dublin, Dublin, Ireland, in 2016. Since then, he was a Research Fellow with the Irish National Telecommunications Research Centre (CONNECT), Trinity College Dublin, Dublin, Ireland, until 2018. He is currently a Lecturer with the University College Dublin, Dublin, Ireland. His areas of research include wireless communications, digital signal processing for communications, multiuser communications, and multicarrier systems. 
Beatrice Cabon received the Ph.D. degree from the Institut Polytechnique Grenoble (Grenoble-INP), Grenoble, France, in 1986, and has been a Professor since 1989. She has been the Head of a research group on microwave-photonics techniques at IMEP-LAHC, Grenoble, France, since 1993. From 1998 to 2008 , she was also the coordinator of the club optics and microwaves of the French Optical Society. She also coordinated two European projects Networks of Excellence funded by the European commission, FP6-IST-2001-32786 NEFERTITI (2002-2005) and FP6-IST-26592 ISIS (2006-2009). Her research interests include microwave photonics, photonic-microwave signal processing, and optical links for high bit rate signals. She has authored or coauthored more than 250 technical publications and is the editor of five books in these areas.
Liam P. Barry received the B.E. degree in electronic engineering and the M.Eng.Sc. degree in optical communications from the University College Dublin, Dublin, Ireland, in 1991 and 1993, respectively. He received the Ph.D. degree from the University of Rennes, France. From February 1993 until January 1996, he was a Research Engineer with the Optical Systems Department of France Telecom's Research Laboratories (now Orange Labs). In February 1996, he joined the Applied Optics Centre, Auckland University, New Zealand, as a Research Fellow and in March 1998, he took up a lecturing position with the School of Electronic Engineering, Dublin City University and established the Radio and Optical Communications Laboratory. He is currently a Professor with the School of Electronic Engineering, a Principal Investigator for Science Foundation Ireland, and the Director of the Radio and Optical Communications. He has published more than 200 articles in internationally peer-reviewed journals, more than 300 papers in international peer-reviewed conferences, and holds ten patents in the area of optoelectronics. He has been a TPC Member for the European Conference on Optical Communications since 2004 and will serve as conference chair for ECOC2019 in Dublin, Ireland. He also served as a TPC Member for the Optical Fiber Communication Conference (OFC), serving as the Chair of the Optoelectronic Devices Subcommittee for OFC 2010. 